\title{
Language Networks: Their Structure, Function, and Evolution
}

\author{
RICARD V. SOLÉ, ${ }^{1,2}$ Bernat COROMINAS-MURTRA, ${ }^{1}$ SERGI VALVERDE, ${ }^{1}$ AND LUC STEEL $\boldsymbol{S}^{3,4}$ \\ ${ }^{1}$ ICREA-Complex Systems Lab, Universitat Pompeu Fabra (GRIB), Barcelona 08003, Spain; ${ }^{2}$ Santa Fe Institute, \\ Santa Fe, New Mexico 87501; ${ }^{3}$ Sony CSL Paris, 6 Rue Amyot, Paris 75005, France; and \\ ${ }^{4}$ Artificial Intelligence Lab, Vrije Universiteit Brussel, Brussels 1050, Belgium
}

Received November 4, 2009; accepted November 29, 2009

\begin{abstract}
Human language is the key evolutionary innovation that makes humans different from other species. And yet, the fabric of language is tangled and all levels of description (from semantics to syntax) involve multiple layers of complexity. Recent work indicates that the global traits displayed by such levels can be analyzed in terms of networks of connected words. Here, we review the state of the art on language webs and their potential relevance to cognitive science. The emergence of syntax through language acquisition is used as a case study to illustrate how the approach can shed light into relevant questions concerning language organization and its evolution. (C) 2010 Wiley Periodicals, Inc. Complexity 15: 20-26, 2010
\end{abstract}

Key Words: language; neural networks; complex networks; syntax; network theory; evolution; phase transitions

\section{INTRODUCTION}

anguage is a privileged window into the mind, since words allow us to compose thoughts and language impairment provides deep clues about brain organization and its decay. It also changes with brain as it develops through the first years of life. Moreover, its evolutionary origins are inseparable from the evolution of cortical maps and both might have actually coevolved [1]. As it happens with the brain connection matrix or connectome [2], language is formed of multiple interacting pieces and levels.

Corresponding author: Ricard V. Solé; ICREA-Complex Systems Lab, Universitat Pompeu Fabra (GRIB), Dr Aiguader 80, 08003 Barcelona, Spain. (e-mail: ricard.sole@upf.edu)
Using Saussure's definition, "language is a system of interdependent terms in which the value of each term results solely from the simultaneous presence of the others" [3]. Looking at them as interacting units would provide us with a picture of its global organization. However, if a cartography of language were possible, how would language maps look like? Is there a connectome for human language? If yes, what could be learned from its origins, acquisition, and universal traits?

From the late 1950s, an intense debate among cognitive scientists changed the mainstream of linguistics, ruling out studies based on statistics and information-theoretic views and focusing research on the underlying, generative rules of language [4, 5]. Generally, the individual as a tabula rasa, who acquires linguistic knowledge only by 
statistical inference, was rejected. This view was tied to a picture of language involving an innate component influencing the set of available rules to generate linguistic structures. Such biological element would pervade the shape and structure of language and its acquisition [6]. Statistical patterns on the other hand revealed the presence of some universal trends, the best known being the so-called Zipf's law [7]. Specifically, if $P(f)$ is the proportion of words whose frequency is $f$ in a given text, Zipf's law is a so-called scaling law:

$$
P(f) \propto f^{-\alpha}
$$

with $\alpha \approx 2$. This means, generally, that most words are rare and a few are very common. Its relevance was questioned on the basis that a randomized model seems to reproduce such frequency distribution [5] although a detailed analysis does not support that view [8]. Actually, several different mechanisms can generate Zipf-like statistics [9] and thus, one needs to be careful in not overinterpreting the meaning of this law. Perhaps the greatest limitation is that it ignores a fundamental ingredient of language structure: the fact that words (or more generally, linguistic units) are interacting entities. As will be shown below, words together with their relationships define new types of statistical patterns that can be described as networks.

The observation of universal trends opens several questions: What is their origin? Can they be explained in terms of natural selection [10] or cultural transmission [11]? The presence of universals has been a matter of discussion within both linguistics and cognitive sciences but only partial understanding has been achieved so far. The answers might come from an interdisciplinary effort, with ideas taken outside from the strict realm of linguistics [12, 13]. In this context, universals are well known in both physics and complexity theory: very different systems made of multiple interacting units can exhibit exactly the same scaling laws if they share similar interaction rules $[14,15]$. These rules are responsible for the large-scale structure of these systems and allow to uncover the underlying generative mechanisms on top of which selection takes place.

In this article, we discuss the value of the study of the patterns and processes underlying language structure from a network perspective. We will argue that their global organization and how they change through acquisition allows to provide the tentative answers to some of the previous questions.

\section{LANGUAGE AS A COMPLEX NETWORK}

Language is a system of interacting units. As such, we can map their relations onto a graph in the hope that such
FIGURE 1
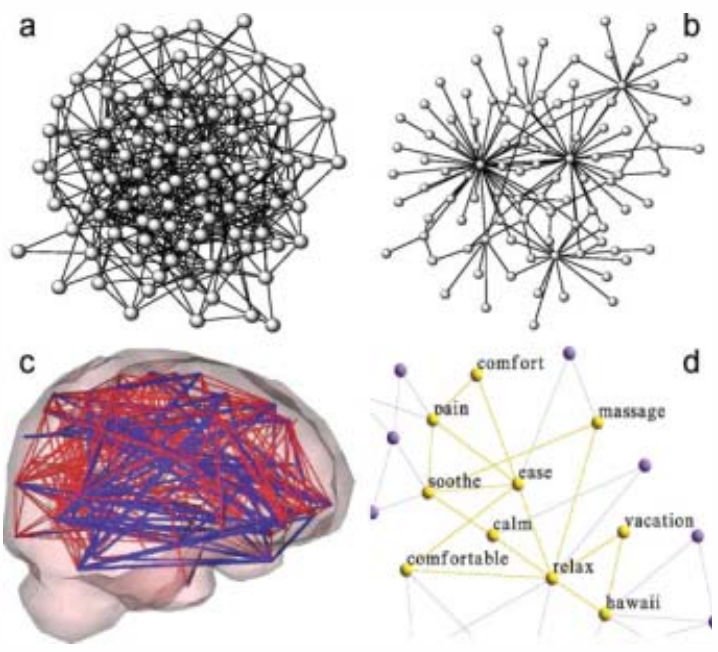

Two main classes of networks have been widely identified in real systems [25, 26], namely random, homogeneous [27] ones (a) and scale-free [28] (b), respectively. In random networks most elements have a number of links around a given average degree $\langle\mathrm{k}\rangle$. This seems to be the standard case for cortical maps [29] (c) and the probability $P(k)$ of finding a node with $k$ links falls off in an exponential fashion, i.e., $P(k)=C \exp (-k / K)$ where $K$ is a characteristic cut-off. Scale-free webs are highly heterogeneous, following power laws, i.e., $\mathrm{P}(\mathrm{k})=\mathrm{Ck}^{-\gamma}$. This would be the case of language graphs, such as semantic or syntactic webs. In (d) we show a small piece of a semantic web where links indicate associations [30]. For a given pair of words such as \{Hawaii and pain\} a short path can be found connecting them. In this case, the path is formed by \{Hawaii, relax, ease, and pain\} with only three links (or degrees of separation) required to reach one from the other. In these webs, paths are typically very short and triangles very frequent, enhancing rapid association between related words. These two features define their small world organization. [Color figure can be viewed in the online issue, which is available at www.interscience.wiley.com.]

mapping will capture fundamental traits of language under a global picture. Such approach has been successful in different fields [16] including ecology [17], computation [18], coding [19], cell and molecular biology [20], neuroscience [21-23] or communication networks [24], revealing novel patterns that can help understanding the structure, robustness, and evolution of these systems. Networks display a limited number of organization patterns (Figure 1) and are not at all new in language studies. At the level of grammar, network-driven approaches include, among others, word grammar [31], which is a global proposal to study language from the network viewpoint, or dependency grammars, [32]. Several nets have been studied, including bipartite graphs based on lexical matrices [33], co-ocurrence [34-36], syntactic [37], semantic [38-42] graphs, and brain activation graphs associated to cognitive tasks involving language [43]. 
Three main features seem to be shared by all language networks. First, they are sparse, meaning that the average number of links per node is small. Second, they have a small world structure. In a small world web, a very large fraction of triangles is found, much larger than expected from randomness. On the other hand, these webs are very well communicated: it is very easy to reach a given element from another one through very short paths [44]. This is measured by means of the so-called path length $D$, which tells us the minimal number of steps required (on average) to reach a given node from any other node. The SW architecture has many implications for optimality as it occurs, for example, with cortical maps [45]. Here, short paths are the fingerprint of efficient information transfer. Pathological states such as Alzheimer's disease [46] display consistently larger path lengths, thus revealing a loss of efficiency. Finally, language networks are highly heterogeneous: Most elements are connected to one or two others and only a handful of them (the hubs) have a very large number of links [Figure 2(a)]. Mathematically, they follow a power law: if $P(k)$ indicates the frequency of elements having $k$ links with others, these systems exhibit a so called scale-free (SF) degree distribution [Figure 2(b)]:

$$
P(k) \propto k^{-\gamma}
$$

with a scaling exponent within the interval $2<\gamma<3$. It can be shown that this interval defines a window of existence of SF nets: outside these bounds the network either becomes easily disconnected $(\gamma>3)$ or too dense $(\gamma<2)$. Networks optimizing communication at low cost (in terms of links) will have larger $\gamma$ values but are more likely to deteriorate with the loss of their hubs [47]. Instead, smaller scaling exponents will allow a larger robustness against the removal of hubs but will also involve a larger number of alternative paths and more connected elements.

Within the context of semantic webs, meaning relations enable us to conceive a network, where links glue interrelated concepts [38-42]. One of the most interesting examples is Wordnet [48] where words are connected through various kinds of semantic links. Despite their large size $\left(N \sim 10^{4}-10^{5}\right)$ the path length in these semantic webs is just $D \approx 3$. The efficient character of the semantic network is tied to an important, universal and yet apparently undesirable property of language: polysemy. Consistently with this view, the analysis of semantic networks reveals a likely reason for polysemy to exist and be so widespread. The answer lies on the global organization of these graphs. The analysis of Wordnet shows a scale-free structure with $P(k) \sim k^{-3}$ thus suggesting it is highly optimized. Here most elements would be more specialized, and thus semantically linked to just a few others. By contrast, a few of them would have a large number of semantic links.
FIGURE 2
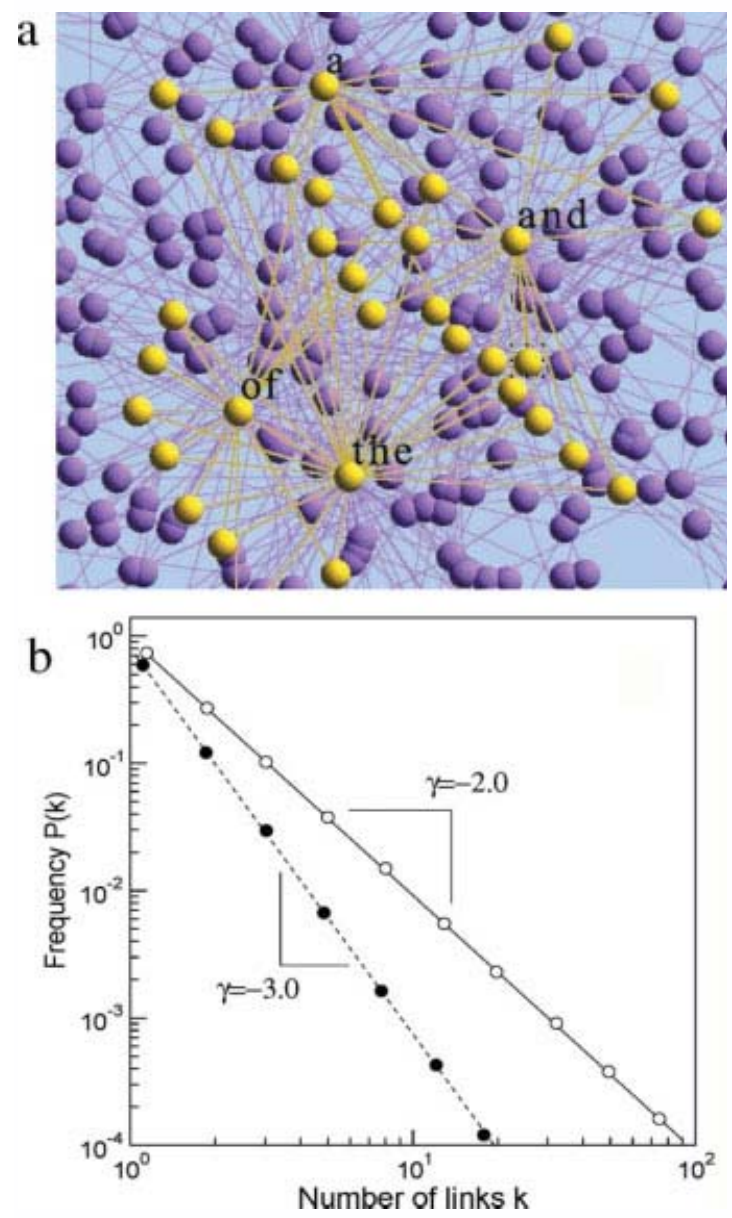

Language networks. Different graphs can be obtained by using different ways of relating words. In (a) the central core of connected words in such a graph is shown, with highly connected hubs (and their connections) shown in yellow. Here, links indicate that words are adjacent to each other in at least one sentence within a given text. The structure of language networks is characterized by a scale-free degree distribution (b) with $P(k) \sim k^{-\gamma}$, with $2 \leq \gamma \leq 3$ (in log-log scale it gives a straight line). Different webs display different, characteristic exponents $\gamma$. In double logarithmic scale, a straight line is obtained, since we have $\log P(k) \approx \gamma \log k$, being $\gamma$ the slope. [Color figure can be viewed in the online issue, which is available at www.interscience.wiley.com.]

Actually, this network is a small world provided that polysemy is included. Additionally, the scale-free topology of semantic webs places some constraints on how these webs can be implemented in neural hardware [39]: The high clustering found in these webs favors search by association, while the short paths separating two arbitrary items makes search very fast [40].

A different class of LNs is obtained from syntactic relations (see Figure 3). Syntax defines the set of rules 


\section{FIGURE 3}

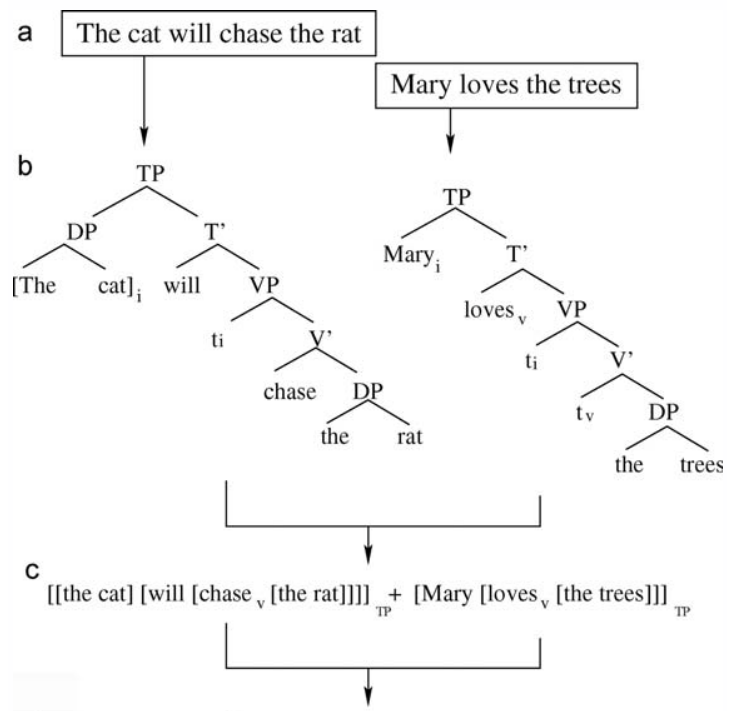

d

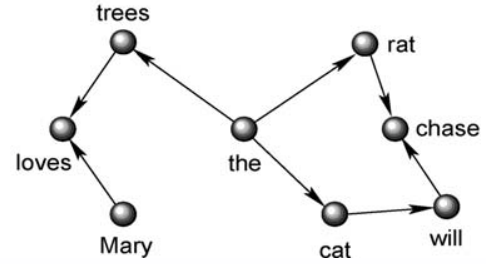

A method to build a syntax network from a corpus. For every sentence (a) we perform the derivation (b). We identify the constituent structure and the main verb in finite form (c). This structure can then be projected and aggregated into syntactic dependencies (d). We assume the head of the phrase to be the semantically most relevant item of the phrase and the main verb the head of the whole sentence. From these dependencies we build a graph. Other ways more sophisticated (including labels for the arcs, for example) can be found in [31].

responsible for building structures [31, 32, 37]. In syntactic webs the hubs correspond to functional words (such as articles or prepositions). In these networks, the scaling exponent has been found to be $\gamma_{\text {syntax }} \approx 2.2$, closer to the lower boundary $\gamma=2$. Paths are very short, involving just 3-4 degrees of separation. The emergence of syntactic organization through language acquisition in children provides a perfect example of how a network perspective can help. This will be developed in detail as a case study in the next section.

\section{THE ONTOGENY OF SYNTAX}

Let us illustrate the power of looking at language on a global basis by studying the problem of syntax develop- ment in children. Language acquisition involves all the topics relevant to our paper: it leads to the emergence of a powerful system of communication (function), through a process of change (evolution) and to a well-organized hierarchy of word relations, the syntax (structure). It has been pointed out that child language (together with ape and pidgin languages) may actually help understanding language origins $[49,50]$.

Children acquire language by properly using phonological, syntactic, and semantic rules in spite that no one teaches them. They can generate a virtually infinite set of grammatically correct sentences after being exposed to a rather limited number of input examples. Moreover, although the lexicon shows a monotonous growth as new words are learned, the pattern of change in syntactic organization involves a shift from babbling, to single words, to the rude two-words grammar to a fully, complex adult grammar [51]. How can children acquire syntax? Are there some specific, basic rules predefined as a part of the biological endowment of humans? Using a global network perspective allows to measure changes in the patterns of word-word relations. It is thus a natural framework for studying how syntax develops. The network approach considers the number of links among words as the key property and thus places dependencies (instead of words) at the center of the analysis. If syntax is a system-level property, it seems reasonable to study its structure and development by means of a global, system-level approach where all observed syntactic relations are considered.

By analyzing child's productions from the CHILDES Database [52-55], a remarkable shift is well appreciated by using a network-level. This is displayed in Figure $4(\mathrm{a}-\mathrm{c})$, where the global architecture of syntactically connected words is shown close to the syntactic spurt. A sharp change is found around 2 years. Right after the transition (b) the network displays all the features found in adult webs, whereas immediately before (a) it does not. Interestingly, the pretransition networks have tree-like structure, very different from the post-transition ones, displaying a very different, small world, and scale-free topology with a stable exponent $\gamma \approx 2.3$. All relevant measures of network complexity exhibit a sharp jump, matching a change in syntactic structure length.

The observed transition occurs in parallel with the emergence of functional particles and inflectional morphology. This phenomenon implies that semantically degenerated items (such as it) which are the hubs in the prefunctional stage are eventually replaced by functional words, absent through the early period. There is also a clear change in the behavior of the particle $a$ (the schwa) acting as a protofunctional, superhub particle just in the transition period. 


\section{FIGURE 4}

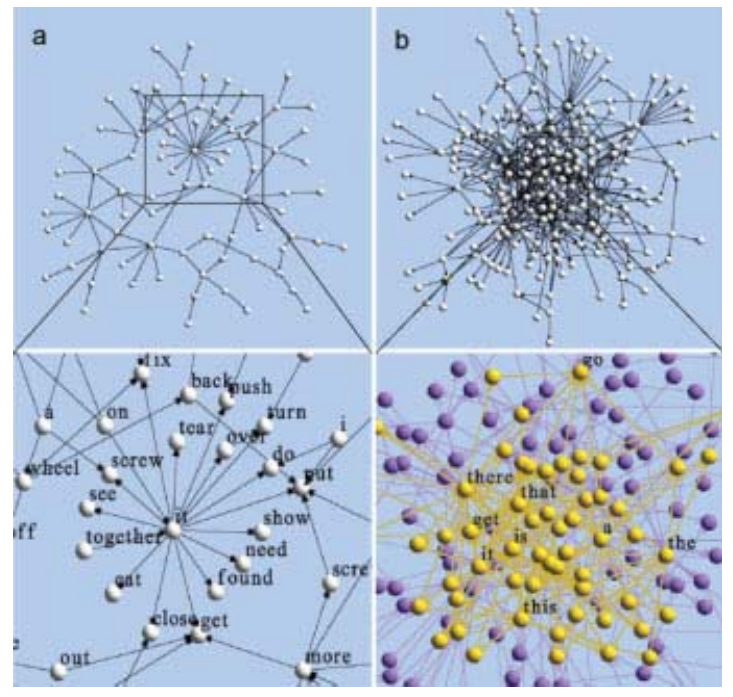

Sudden changes in the global organization of children syntactic networks. Two networks are shown: here (a) is obtained at the presyntactic spurt whereas (b) is observed right after 2 years (these are separated by 1 month around the age of two). A clear transition occurs from a tree-like structure to a scale-free, small world syntax graph. The lower row shows zoomed subsets of each network. Dominating hubs prior and after the two-year transition are very different, thus indicating a deep reorganization. Networks have been reconstructed from the CHILDES database using Peter's corpora [53-55]. [Color figure can be viewed in the online issue, which is available at www.interscience.wiley.com.]

What is the meaning of these sharp changes? Sudden transitions in growing graphs are known to occur in random networks with increasing numbers of links [56]. In these models, a threshold phenomenon known as percolation leads to a global change where the web shifts from a set of many disconnected small graphs into a web with a large component of connected elements. However, these networks are very different from those seen in children graphs and no hub rearrangements are at work. Similarly, models based on rich-gets-richer mechanisms [57] where nodes having more links tend to acquire further connections also fail to reproduce neither the transition nor the hub changes. The observed shifting pattern is thus hard to explain in terms of a model of network self-organization, as suggested by some studies [58-60]. Instead, hub rearrangements suggest the presence of internal constrains, pointing toward some kind of hardwired element. This example provides a good illustration of the power of the network approximation and reveals a facet of language that is not shared by any other class of complex network.

\section{DISCUSSION}

Two considerations emerge from our analysis. First, it points to new types of universal features of language beyond Zipf's law. These potential universals do not focus on properties of the elements in language inventories or specific grammar rules but rather on global, quantitative patterns that include a combinatorial element. The case study presented here opens new questions that will require careful inspection. If standard models of network evolution are unable to explain the observed pattern of acquisition, new models will be needed. This leads us to look towards another useful perspective: the use of artificial systems where complex agents can communicate and evolve grammatical rules.

Embodied, robotic agents (Figure 5) provide an alternative approach to test the presence of universals. Current work in the fields of evolved artificial languages [61-64] and embodied communicating agents [65, 66]

\section{FIGURE 5}

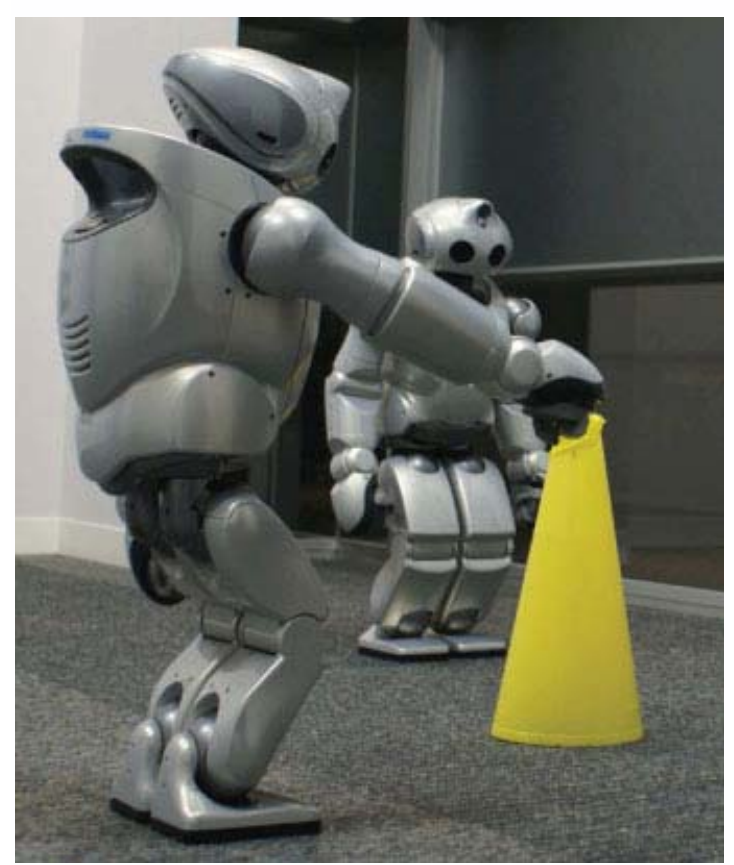

The search for universals in language might strongly benefit from the analysis of artificial agents. New robotic implementations, such as the Qrio robots shown here, will allow researchers to study the emergence of syntax in nonbiological scenarios. These robots are able to explore their environment, locate and name objects and exchange their sharted knowledge with other robots. Picture provided with permission by SONY Computer Science Lab. [Color figure can be viewed in the online issue, which is available at www. interscience.wiley.com.] 
indicates that lexicon but also rudimentary forms of grammar can emerge in artificial systems [67]. The observed patterns of network organization displayed in child development and their meaning could be explored by looking at the emergence of complex combinatorial rules in such evolving artificial grammars. In this context, fluid construction grammars (FCG) can be used in order to analyze the emergence of language under our network perspective [68]. Using FCGs it will be possible to know whether the sudden reorganization of syntax graphs requires a biological substrate to be explained. Alternatively, it might instead result from a universal mechanism of combinatorial explosion. If so, innatism would be seriously flawed. On the other hand, there exists also the possibility that the acquisition of complex grammars can take place following different paths with no common universals. In this context, artificial systems offer a third avenue, between real network data and mathematical models of graphs, to gain insight into these deep questions.

\section{Questions for Future Research}

- Are there statistical differences among networks for different languages?

- Can artificial communities of agents develop languages with scale-free network structures?

- How are different language networks modified through aging and brain damage?

- Is there a link between cortical maps involved in language and observed language networks?

- How can neural models of language development generate the observed language webs?

\section{Acknowledgments}

The authors thank Guy Montag, M. Christiansen, P. Villoslada, D. Krakauer, M. Gell-Mann, and the members of the CSL for useful comments. This work has been supported by grants FIS2004-0542, IST-FET ECAGENTS EU 011940, by the Santa Fe Institute and the Sony Computer Science Laboratory.

\section{REFERENCES}

1. Deacon, T.W. The Symbolic Species: The Co-Evolution of Language and the Brain; Norton: New York, 1997.

2. Sporns, O.; Tononi, G.; Kötter, R. The human connectome: A structural description of the human brain. PLos Comput Biol 2005, 1, 245-251.

3. de Saussure, F. Course of General Linguistics; Payot: Lausanne, 1959.

4. Miller, G.A. The cognitive revolution: A historical perspective. Trends Cogn Sci 2003, 7, 141-144.

5. Miller, G.A.; Chomsky, N. Finitary models of language users. In: Handbook of Mathematical Psychology, Vol. 2; Luce, R.D., Bush, R.R.; Galanter, E., Eds., Mouton: The Hague; 1963.

6. Chomsky, N. Syntactic Structures; Mouton: The Hague, 1957.

7. Zipf, G.K. Human Behavior and the Principle of Least-Effort; Addison-Wesley: Cambridge, MA, 1949.

8. Ferrer Cancho, R.; Solé, R.V. Zipf's law in random texts. Adv Complex Syst 2002, 5, 1-6.

9. Newman, M.E.J. Power laws. Pareto distributions and Zipf's law. Contemp Phys 2005, 46, 323-351.

10. Nowak, M.A.; Komarova, N.L. Towards an evolutionary theory of language. Trends Cogn Sci 2001, 5, $288-295$.

11. Kirby, S.; Dowman, M.; Griffiths, T.L. Innateness and culture in the evolution of language. Proc Natl Acad Sci USA 2007, 104, 5241-5245.

12. Christiansen, M.H.; Kirby, S. Language evolution: Consensus and controversies. Trends Cogn Sci 2003, 7, 300-307.

13. Érdi, P. Complexity underestimated? Behav Brain Sci 2003, 26, 676-677.

14. Kauffman, S. The Origins of Order: Self-Organization and Selection in Evolution; Oxford University Press: Oxford, 1993.

15. Solé, R.V.; Goodwin, B.C. Signs of Life: How Complexity Pervades Biology; Basic Books, Perseus: New York, 2001.

16. Solé, R.V.; Ferrer Cancho, R.; Montoya, J.M.; Valverde, S. Selection, tinkering, and emergence in complex networks. Complexity 2002, 8, 20-33.

17. Montoya, J.M.; Pimm, S.A.; Solé, R.V. Ecological networks and their fragility. Nature 2006, 442, 259-264.

18. Valverde, S.; Ferrer Cancho, R.; Solé, R.V. Scale free networks from optimal design. Europhys Lett 2002, 60, $512-517$.

19. Kim, J.-H.; Ko, Y.-J. Error-correcting codes on scale-free networks. Phys Rev 2004, 69, 067103.

20. Solé, R.V.; Pastor-Satorras, R.Complex networks in genomics and proteomics. In: Handbook of Graphs and Networks; Bornholdt, S.; Schuster, H.G., Eds.; Wiley: Berlin, 2002; pp 147-169.

21. Eguiluz, V.; Cecchi, G.; Chialvo, D.R.; Baliki, M.; Apkarian, A.V. Scale-free brain functional networks. Phys Rev Lett 2005, 92, 018102.

22. Sporns, O.; Chialvo, D.R.; Kaiser, M.; Hilgetag, C.C. Organization, development, and function of complex brain networks Trends Cogn Sci 2004, 8, 418-425.

23. He, Y.; Chen, Z.-J.; Evans, A.C. Small-world anatomical networks in the human brain revealed by cortical thickness from MRI. Cereb Cortex 2007, 17, 2407-2419.

24. Albert, R.; Jeong, H.; Barabási, A. Diameter of the world wide web. Nature 1999, 401, 130-131.

25. Dorogovtsev, S.N.; Mendes J.F.F. Evolution of Networks: From Biological Nets to the Internet and WWW; Oxford University Press: Oxford, 2003.

26. Boccaletti, S.; Latora, V.; Moreno, Y.; Chavez, M.; Hwang, D.-U. Complex networks: Structure and dynamics. Phys Rep 2006, 424, 175-308. 
27. Bollobas, B. Random Graphs; Academic Press: London, 1985.

28. Caldarelli, G. Scale-Free Networks. Complex Webs in Nature and Technology; Oxford University Press: New York, 2007.

29. Kaiser, M. Brain architecture: A design for natural computation. Philos Trans R Soc A 2007, 365, 3033-3045.

30. Steyvers, M.; Tenenbaum, J.B. Graph theoretic analyses of semantic networks: Small worlds in semantic networks. Cogn Sci 2005, 29, 41-78.

31. Hudson, R. Language Networks. The New Word Grammar; Oxford University Press: Oxford, 2007.

32. Melçuck, I. Dependency Syntax: Theory and Practice; State University of New York: Albany, 1989.

33. Niyogi, P. The Computational Nature of Language Learning and Evolution; MIT Press: Cambridge, MA, 2006.

34. Ferrer i Cancho, R.; Solé, R.V. The small-world of human language. Proc R Soc Lond Ser B 2001, 268, 2261-2266.

35. Ke, J. Selforganization and language evolution: System, population, and individual. PhD thesis. City University of Hong Kong, 2004.

36. Masucci, A.P.; Rodgers, G.J. Network properties of written human language. Phys Rev E 2006, 74, 026102.

37. Ferrer Cancho, R.; Koehler, R.; Solé, R.V. Patterns in syntactic dependency networks. Phys Rev E 2004, 69, 32767.

38. Sigman, M.; Cecchi, G.A. Global organization of the Wordnet lexicon. Proc Natl Acad Sci USA 2002, 99, 1742-1747.

39. Steyvers, M.; Tenenbaum, J.B. The large-scale structure of semantic networks: Statistical analyses and a model of semantic growth. Cogn Sci 2005, 29, 41-78.

40. Motter, A.E., de Moura, A.P.S., Lai, Y-C., Dasgupta, P. Topology of the conceptual network of language. Phys Rev E 2002, 65, 065102.

41. Kinouchi, O.; Martinez, A.S.; Lima, G.F; Lourenço, G.M.; Risau-Gusman, S. Deterministic walks in random networks: An application to thesaurus graphs. Phys A 2002, 315, 665-676.

42. Holanda, A.J., Torres Pisa, I., Kinouchi, O., Souto, A, Seron Ruiz, E. Thesaurus as a complex network. Phys A 2004, 344, 530536.

43. Pulvermuller, F. Brain reflections of words and their meaning. Trends Cogn Sci 2001, 5, 517-524.

44. Watts, D.J.; Strogatz, S.H. Collective dynamics of 'small-world' networks. Nature 1998, 393, 440-442.

45. Wen, Q.; Chklovskii, D.B. Segregation of the brain into gray and white matter: A design minimizing conduction delays. PLoS Comp Biol 2005, 1, 617-630.

46. Chan, A.S.; Butters, N.; Salmon, D.P. The deterioration of semantic networks in patients with Alzheimer's disease: A crosssectional study. Neuropsychologia 1997, 35, 241-248.

47. Albert, A.; Hawoong, J.; Barabási, A.L. Error and attack tolerance of complex networks. Nature 2000, 406, 378-382.

48. http://wordnet.princeton.edu/.

49. Maynard-Smith, J.; Szathmàry, E. The Major Transitions in Evolution; Oxford University Press: New York, 1995.

50. Bickerton, D. Language and Species; Chicago University Press: Chicago, 1990.

51. Radford, A. Syntactic Theory and the Acquisition of English Syntax: The Nature of Early Child Grammars in English; Oxford: Blackwell, 1990.

52. Corominas-Murtra, B.; Valverde, S.; Solé, R.V. The ontogeny of scale-free syntax networks. Evidence for a phase transition in early language acquisition. Adv Complex Syst 2009, 12, 371-392.

53. MacWhinney, B. The CHILDES Project: Tools for Analyzing Talk, 3rd ed.; Lawrence Erlbaum Associates: Mahwah, NJ, 2000.

54. Bloom, L.; Hood, L.; Lightbown, P. Imitation in language development: If, when, and why. Cogn Psychol 1974, 6, 380-420.

55. Bloom, L.; Hood, L.; Hood, L. Structure and variation in child Language. Monog Soc Res Child Dev 1975, 40, serial No. 160.

56. Erdös, P.; Rényi, A. On random graphs. Publicationes Mathematicae (Drebecen) 1959, 6, $290-297$.

57. Barabási, A.L.; Albert, A. Emergence of scaling in random networks. Science 1999, 286, 509-512.

58. Ferrer Cancho, R. When language breaks into pieces: A conflict between communication through isolated signals and language. Biosystems 2006, 84, 242-253.

59. Ferrer Cancho, R.; Bollobás, R.; Riordan, O. The consequences of Zipf's law for syntax and symbolic reference. Proc R Soc Lond Ser B 2005, 272, 561-565.

60. Solé, R.V. Syntax for free? Nature 2005, 434, 289.

61. Tonkes, B.; Blair, A.; Wiles, J. Evolving learnable languages. In: Advances in Neural Information Processing Systems 12; Solla, S.A.; Leen, T.K., Muller, K.-R., Eds., MIT Press, 2002; pp 66-72.

62. Cangelosi A.; Parisi D., Eds. Simulating the Evolution of Language; Springer: London, 2002.

63. Kirby, S. Natural language from artificial life. Artif Life 2002, 8, 185-215.

64. Smith, K.; Kirby, S.; Brighton, H. Iterated learning: A framework for the emergence of language. Artif Life 2003, 9, 371-386.

65. Steels, L. Evolving grounded communication for robots. Trends Cogn Sci 2003, 7, 308-312.

66. Floreano, D.; Mitri, S.; Magnenat, S.; Keller, L. Evolutionary conditions for the emergence of communication in robots. Curr Biol 2007, 17, 514-519.

67. Steels, L. The emergence and evolution of linguistic structure: From lexical to grammatical communication systems. Connect Sci 2005, 17, 213-230.

68. http://www.emergent-languages.org/fcg/index.html. 